\title{
Progress in Lattice QCD
}

\author{
Yoshinobu Kuramashi; ${ }^{* a, b, c}$ \\ ${ }^{a}$ Graduate School of Pure and Applied Sciences and Center for Computational Sciences, \\ University of Tsukuba, Tsukuba, Ibaraki 305-8571, Japan \\ ${ }^{b}$ Center for Computational Sciences, University of Tsukuba, Tsukuba, Ibaraki 305-8577, Japan \\ ${ }^{c}$ RIKEN Advanced Institute for Computational Science, \\ 7-1-26 Minatojima-minami-machi, Chuo-ku, Kobe 650-0047, Japan \\ E-mail: kuramasiahet.ph.tsukuba.ac.jp
}

It has been believed that lattice QCD is an ideal tool to investigate the nonperturbative dynamics of strong interactions from first principles. In order to fullfill the promise a lot of effort has been devoted to control the systematic errors to increase reliability. We explain what kind of systematic errors still remain in current lattice QCD simulations. Another important issue is the recent progress in phase shift analysis of hadron-hadron interactions and direct investigation of multi-nucleon systems based on QCD. We discuss the $\rho-\pi \pi$ resonance and the nuclei with small mass number.

35th International Conference of High Energy Physics - ICHEP2010,

July 22-28, 2010

Paris France

${ }^{*}$ Speaker. 


\section{Introduction to lattice QCD}

Lattice QCD is defined by the path-integral formalism on the discretized four-dimensional space time. Physical quantities are extracted from Green functions with appropriate choices of operators, which are expressed as

$$
\left\langle\mathscr{O}\left[U_{\mu}, q, \bar{q}\right]\right\rangle=\frac{1}{Z} \int \mathscr{D} U_{\mu} \mathscr{D} q \mathscr{D} \bar{q} \mathscr{O}\left[U_{\mu}, q, \bar{q}\right] \mathrm{e}^{-S_{\mathrm{QCD}}^{L}\left[U_{\mu}, q, \bar{q}\right]},
$$

where $S_{\mathrm{QCD}}^{L}$ represents the lattice QCD action. $U_{\mu}$ is the link variable as a function of the gauge field and $q$ and $\bar{q}$ denote the quark and anti-quark fields, respectively.

Numerical technique with Monte Carlo method allows us to evaluate the Green functions nonperturbatively up to statistical errors which can be arbitrarily reduced according to $1 / \sqrt{N}$ with $N$ the number of independent configurations (Monte Carlo samples). We have three sets of physical parameters: lattice size $\left(N_{x}, N_{y}, N_{z}, N_{t}\right)$, bare coupling $g$, which controls the lattice spacing $a$, and quark masses $\left(m_{\mathrm{u}}, m_{\mathrm{d}}, m_{\mathrm{s}}, \ldots\right)$. There are four major systematic errors in lattice QCD calculations: (i) finite volume effects, (ii) finite lattice spacing effects, (iii) quenched approximation in which the vacuum polarization effects are neglected and (iv) chiral extrapolation of up and down quark masses. It is rather straightforward to diminish the first and the second errors with the use of larger and finer lattices. We already know how to incorporate the vacuum polarization effects of up, down and strange quarks to remove the quenching errors. Direct simulation at the physical values of up and down quark masses can avoid any possible systematic uncertainties associated with their chiral extrapolation from unphysically heavy simulation values, where the computational cost is cheap, to the physical ones. The reduction of the systematic errors, however, demands enormous computational cost. An expected computational cost is roughly described by

$$
\operatorname{cost} \propto(4 \text { dim. physical volume })^{5 / 4} \cdot(\text { lattice spacing })^{-6 \sim-7} \cdot(\text { quark mass })^{-2 \sim-3},
$$

where we assume the Hybrid Monte Caro algorithm to incorporate the vacuum polarization effects.

\section{Light hadron spectrum}

Light hadron spectrum is one of the most fundamental quantities both in physical sense and technical one. On the physical side we can check the validity of QCD by investigating whether or not lattice QCD calculations successfully reproduce the light hadron spectrum with the choice of a couple of physical inputs to fix the quark masses and lattice spacing. On the technical side the hadron masses are extracted from hadron two-point function that is the simplest Green function consisting of quark fields: $\left\langle\mathscr{O}_{h}(t) \mathscr{O}_{h}^{\dagger}(0)\right\rangle \stackrel{t \gg 0}{\sim} Z \exp \left(-m_{h} t\right)$. Meson operators are made of the quark and antiquark fields to represent the correct quantum numbers. Three quark fields are used for baryon operators.

For almost twenty years after the first lattice QCD calculation of the hadron masses in 1981[1], most of the large-scale simulations were carried out in the quenched approximation. A primary reason is that the inclusion of the vacuum polarization effects requires $O\left(10^{2}\right)$ times as much computational cost as the quenched approximation. In the late 90s the PACS-CS collaboration performed 


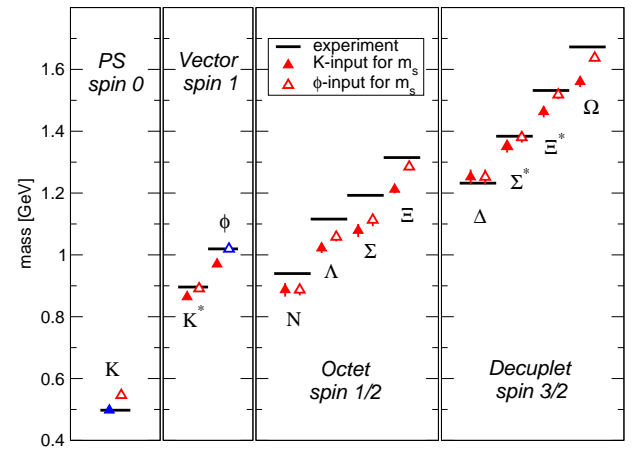

Figure 1: Quenched light hadron spectrum compared with experiment [2].

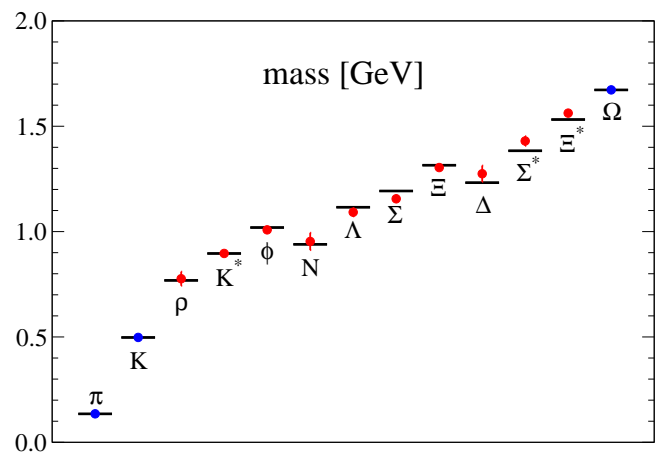

Figure 2: Light hadron spectrum in 2+1 flavor QCD with $m_{\pi}, m_{K}, m_{\Omega}$ (blue) as physical inputs 3.

a detailed investigation of the quenching effects [2]. The systematic study of the light hadron spectrum in the quenched approximation with other systematic errors under control reveals that the results deviate from the experimental values at a $10 \%$ level. The comparison is depicted in Fig. 10 where the three physical inputs are chosen to be $m_{\pi}, m_{\rho}, m_{K}$ (closed triangles) or $m_{\pi}, m_{\rho}, m_{\phi}$ (open triangles) to determine the averaged up-down quark mass, the strange one and the lattice spacing $a$. The confirmation of the discrepancy between the quenched results and the experimental values drove us to embark on the $2+1$ flavor QCD simulations where the vacuum polarization effects of the up, down and strange quarks are incorporated with an artificial condition $m_{\mathrm{u}}=m_{\mathrm{d}}$. Figure 2 shows one of the recent $2+1$ flavor QCD results for the light hadron spectrum obtained by the PACS-CS Collaboration[3]. Most of the results are consistent with experimental values within error bars, though some cases show $2-3 \%$ deviations at most. Similar results are obtained by other groups [4].

Although there is no doubt that we are successfully reducing the systematic errors, the current situation is not sufficient. The reason is that we find two types of problems in Fig. 2. First one is the artificial isospin symmetry $m_{\mathrm{u}}=m_{\mathrm{d}}$ which is employed in almost all the current simulations due to an algorithmic reason. If we want to discuss the errors at a level of $1 \%$, it is necessary to incorporate the isospin symmetry breaking effects: up-down quark mass difference and also electromagnetic interactions. For the latter there are a couple of exploratory studies to investigate the effects at the unphysically heavy quark mass region[5]. Note that both effects are so small that the investigation at the physical point is highly desired to avoid any contamination associated with chiral extrapolations. Second problem is unstable particles. Some hadrons listed in Fig. 2 are the resonance states in the strong interactions. Although it has been known for a long time that the resonance states should be treated in a proper manner on the lattice [6, it is quite recently that it is made possible. In the next section we will explain the current status of lattice QCD calculation of the $\rho-\pi \pi$ resonance.

\section{3. $\rho-\pi \pi$ resonance}

Phase shift is an essential ingredient to investigate hadron-hadron interactions. In case of the $\rho \rightarrow \pi \pi$ decay the relevant quantity is the $P$-wave scattering phase shift for the $I=1$ two- 
pion system. To study the $\rho$ decay on the lattice we have both theoretical and practical problems. The former was already addressed by Lüscher, who provided a finite size formula relating the energy shift due to the hadron-hadron interactions in a finite box to the phase shift [6]. The latter is a kinematical condition on the lattice: At least we need to satisfy the condition $m_{\rho}>2 m_{\pi}$ incorporating the vacuum polarization effects of the up and down quarks. It is rather recently that 2 $\left(m_{\mathrm{u}}=m_{\mathrm{d}}\right)$ and $2+1\left(m_{\mathrm{u}}=m_{\mathrm{d}} \neq m_{\mathrm{s}}\right)$ flavor simulations at sufficiently light quark masses satisfying the above condition are realized. After the CP-PACS Collaboration carried out the first lattice QCD study of the $\rho$ meson decay width based on the phase shift in 2007[7], several groups are now actively working on it [8].

There are three steps to determine the phase shift in the $I=1 \pi \pi$ system. Firstly, we choose the $\pi \pi$ kinematics such that the invariant mass $\sqrt{s}$ is sufficiently close to the $\rho$ meson mass. Here is one technical problem. In current lattice QCD simulations, where typical spatial extent of the lattice is $2-3 \mathrm{fm}$, the energy of two pions with finite momentum is much larger than the $\rho$ meson mass $2 \sqrt{m_{\pi}^{2}+p^{2}}>m_{\rho}$, where the momentum is discretized as $\vec{p}=(2 \pi / L) \vec{n}$ with ${ }^{\exists} \vec{n} \in \mathbb{Z}^{3}$ in an $L^{3}$ box neglecting the interaction. This difficulty, however, is avoided by considering the moving frame which is a system with a nonzero total momentum. A simplest case is $\sqrt{m_{\pi}^{2}+p^{2}}+m_{\pi}$ for the two free pions and $\sqrt{m_{\rho}^{2}+p^{2}}$ for the $\rho$ meson. This can reduce the invariant mass of the free two pions. The extension of the Lüscher's formula to the moving frame is given in Ref. [9]. Secondly, we extract two energy eigenvalues from $2 \times 2$ correlation matrix:

$$
\left(\begin{array}{cc}
\left\langle\mathscr{O}_{\pi \pi}(t) \mathscr{O}_{\pi \pi}^{\dagger}(0)\right\rangle & \left\langle\mathscr{O}_{\pi \pi}(t) \mathscr{O}_{\rho}^{\dagger}(0)\right\rangle \\
\left\langle\mathscr{O}_{\rho}(t) \mathscr{O}_{\pi \pi}^{\dagger}(0)\right\rangle & \left\langle\mathscr{O}_{\rho}(t) \mathscr{O}_{\rho}^{\dagger}(0)\right\rangle
\end{array}\right) \stackrel{\text { diagonalize }}{\longrightarrow}\left(\begin{array}{cc}
Z_{1} \exp \left(-W_{1}\left(k_{1}\right) t\right) & 0 \\
0 & Z_{2} \exp \left(-W_{2}\left(k_{2}\right) t\right)
\end{array}\right)
$$

where $W$ denotes the energy in the moving frame and $k=|\vec{k}|$ is the associated momentum. The relation between $W$ and $k$ is given by $\sqrt{s}=\sqrt{W^{2}-p^{2}}$ with $k^{2}=s / 4-m_{\pi}^{2}$. An important point is that $\vec{k}$ is not discretized as $(2 \pi / L) \vec{n}$ with ${ }^{\exists} \vec{n} \in \mathbb{Z}^{3}$ anymore because of the interaction effects. Note that the hadron three- and four-point functions of $\left\langle\mathscr{O}_{\pi \pi}(t) \mathscr{O}_{\pi \pi}^{\dagger}(0)\right\rangle,\left\langle\mathscr{O}_{\pi \pi}(t) \mathscr{O}_{\rho}^{\dagger}(0)\right\rangle,\left\langle\mathscr{O}_{\rho}(t) \mathscr{O}_{\pi \pi}^{\dagger}(0)\right\rangle$ yield more complicated quark line diagrams than that of the hadron two-point function of $\left\langle\mathscr{O}_{\rho}(t) \mathscr{O}_{\rho}^{\dagger}(0)\right\rangle$. This is one of major practical reasons that calculation for the $\rho-\pi \pi$ resonance is more difficult than that for the stable hadrons. In the final step the phase shift at the momentum $k$ is obtained by the Lüscher's finite size formula.

It may be intuitively clear to extract the $\rho$ meson decay width by fitting the phase shift data on the lattice with the Breit-Wigner formula. With the present computational resources, however, we are forced to work with the heavier quark masses than the physical values. Since the decay width strongly depends on the kinematical factors at the quark mass where our calculations are carried out, we take different approach.

The $\rho \rightarrow \pi \pi$ decay is phenomenologically described by the interaction lagrangian $\mathscr{L}_{\text {int }}=$ $g_{\rho \pi \pi} \varepsilon_{a b c} \rho_{\mu}^{a} \pi^{b} \partial^{\mu} \pi^{c}$, where $\rho_{\mu}^{a}$ and $\pi^{a}$ are effective fields for the $\rho$ meson and the pion, respectively, with canonical kinetic terms. In terms of the effective $\rho \rightarrow \pi \pi$ coupling constant $g_{\rho \pi \pi}$, the resonance behavior of the $\pi \pi$ phase shift is given by

$$
\tan \delta(k)=\frac{g_{\rho \pi \pi}^{2}}{6 \pi} \frac{k^{3}}{\sqrt{s}\left(m_{\rho}^{2}-s\right)}
$$


with $m_{\rho}$ the resonance mass. We have two parameters $m_{\rho}$ and $g_{\rho \pi \pi}$ to be determined by fitting the lattice data. The decay width at the simulation point is obtained by

$$
\Gamma=\frac{g_{\rho \pi \pi}^{2}}{6 \pi} \frac{k^{3}}{m_{\rho}^{2}} .
$$

However, the result for $\Gamma$ cannot be directly compared with the experimental value of the decay width. The kinematics on the lattice is completely different from the real world because of the heavier quark masses. Instead, assuming the mild quark mass dependence of $g_{\rho \pi \pi}$ we compare the lattice results for $g_{\rho \pi \pi}$ and "the physical value" of $g_{\rho \pi \pi}^{\mathrm{ph}}=5.98(2)$ obtained from $\Gamma^{\mathrm{ph}}=149.1(8)$ $\mathrm{MeV}$ with the experimental values of $m_{\rho}^{\mathrm{ph}}$ and $m_{\pi}^{\mathrm{ph}}$ and $\left(k^{\mathrm{ph}}\right)^{2}=\left(m_{\rho}^{\mathrm{ph}}\right)^{2} / 4-\left(m_{\pi}^{\mathrm{ph}}\right)^{2}$. Table 1 summarizes the recent results for $g_{\rho \pi \pi}$ based on the $\pi \pi$ phase shift [7 [8]. An encouraging observation is that all the results scatter around the physical value, though the errors are rather large.

Table 1: Results for the effective $\rho \rightarrow \pi \pi$ coupling constant $g_{\rho \pi \pi}$.

\begin{tabular}{cccc}
\hline collab. & \#flavor & $m_{\pi}[\mathrm{MeV}]$ & $g_{\rho \pi \pi}$ \\
\hline CP-PACS & 2 & 330 & $6.25(67)$ \\
QCDSF & 2 & $240-810$ & $5.3_{-1.5}^{+2.1}$ \\
ETM & 2 & $290,330,420,480$ & $6.77(67), 6.31(87), 6.19(42), 6.46(40)$ \\
PACS-CS & $2+1$ & 410 & $5.24(51)$ \\
BMW & $2+1$ & 200,340 & $5.5(2.9), 6.6(3.4)$ \\
\hline
\end{tabular}

\section{Nuclei from lattice QCD}

The atomic nuclei have been investigated by treating protons and neutrons as effective degrees of freedom at the nuclear energy scale of a few $\mathrm{MeV}$. At present we know for certain that protons and neutrons are made of quarks and gluons which are more fundamental degrees of freedom. It is a great challenge to reveal the hierarchical structure and property of nuclei based on the first principle of QCD. After an exploratory study of the nucleon-nucleon $(N N)$ system in the mid 90s [10], important steps were recently made with two different kinds of approaches: determination of nuclear force [11] and direct construction of helium nuclei[12] in lattice QCD.

\subsection{Nuclear force}

The authors in Ref. [11] proposed a method to extract a $N N$ potential in lattice QCD. The potential is defined by the equal-time Nambu-Bethe-Salpeter (NBS) wave function

$$
\phi_{E}(\vec{r})=\langle 0|N(\vec{x}+\vec{r}, \overrightarrow{0}) N(\vec{x}, \overrightarrow{0})| B=2, E\rangle,
$$

where $|B=2, E\rangle$ is an eigenstate of QCD with the baryon number 2 and the energy $E$. The local nucleon operator $N(x)$ is constructed from the up and down quark fields $u(x)$ and $d(x)$. The proton operator is defined as $p_{\alpha}(x)=\varepsilon_{a b c}\left(\left[u_{a}(x)\right]^{t} C \gamma_{5} d_{b}(x)\right) u_{c}^{\alpha}(x)$ where $C=\gamma_{4} \gamma_{2}$ and $\alpha$ and $a, b, c$ are the Dirac index and the color indices, respectively. The neutron operator $n_{\alpha}$ is obtained by replacing $u_{c}^{\alpha}$ 
by $d_{c}^{\alpha}$ in the proton operator. The non-local potential $U\left(\vec{r}, \overrightarrow{r^{\prime}}\right)$ is defined by an effective Schrödinger equation for the NBS wave function

$$
-\frac{\Delta}{2 \mu} \phi_{E}(\vec{r})+\int d^{3} r^{\prime} U\left(\vec{r}, \vec{r}^{\prime}\right) \phi_{E}\left(\vec{r}^{\prime}\right)=E \phi_{E}(\vec{r})
$$

where $\mu \equiv m_{N} / 2$ and $E$ are the reduced mass of the nucleon and the nonrelativistic energy, respectively. The velocity (derivative) expansion of $U$ is expressed as $U\left(\vec{r}, \overrightarrow{r^{\prime}}\right)=V(\vec{r}, \nabla) \delta^{3}\left(\vec{r}-\overrightarrow{r^{\prime}}\right)$ with

$$
V(\vec{r}, \nabla)=V_{C}(r)+V_{T}(r) S_{12}+V_{L S}(r) \vec{L} \cdot \vec{S}+O\left(\nabla^{2}\right) .
$$

Here $S_{12}=3\left(\vec{\sigma}_{1} \cdot \vec{r}\right)\left(\vec{\sigma}_{1} \cdot \vec{r}\right) / r^{2}-\vec{\sigma}_{1} \cdot \vec{\sigma}_{2}$ is the tensor operator with $\vec{\sigma}_{i} / 2$ the spin of $i$-th nucleon, $\vec{S}$ is the total spin operator and $\vec{L}$ the relative angular momentum operator. Note that the first and second terms in the right hand side are the leading order contributions and the third term is the next leading order one.

In the phenomenological analysis of the $N N$ scattering phase shift the solution of the Schrödinger equation with parametrized $N N$ potential is compared with the experimental data. On the other hand, calculation of $\phi_{E}$ on the lattice for various $E$ allows us to determine the nonlocal potential $U$ from Eq. (4.2). However, it is practically difficult to change $E$ with a good resolution, because the momentum is discretized due to the finite lattice size. This problem may be avoided by focusing on the leading term in the derivative expansion of Eq. 4.3) at low energies. The central potential $V_{C}(r)$ at fixed $E$ is extracted as $V_{C}(r)=E+\Delta \phi_{E}(r) / \phi_{E}(r) /(2 \mu)$, where $E$ is obtained from the Green function $G(\vec{r} ; E)$ as a solution of the Helmholtz equation on the lattice[13]. An exploratory study of the central $N N$ potentials at rather heavy quark mass in quenched QCD successfully reproduced empirically known features of the potential [11]: a strong repulsive core at short distances surrounded by an attractive well at medium and long distances. In this conference Aoki, who is one of the authors in Ref. [11], reported recent developments on the potential calculations [14]. They have now succeeded in extracting the tensor potential using the ${ }^{3} S_{1}$ and ${ }^{3} D_{1}$ components mixed by the tensor operator. The potential method is currently applied to investigate the hyperon interactions in 2+1 flavor QCD.

\subsection{Direct construction of helium nuclei}

There are two major technical difficulties in treating the multi-nucleon systems on the lattice. First one is discrimination between the bound state and the scattering state. The binding energy $\Delta E$ of the nucleus, consisting of $N_{N}$ nucleons with the mass $m_{N}$, is very tiny compared with the mass $M$ of the nucleus: $\Delta E / M \sim O\left(10^{-3}\right)$ with $\Delta E=N_{N} m_{N}-M$. This causes a complicated situation where it is difficult to distinguish the physical binding energy from the energy shift due to the finite volume effect in the attractive scattering system[15]. One way to solve the problem is to investigate the volume dependence of the measured energy shift: In the attractive scattering system, the energy shift is proportional to $1 / L^{3}$ at the leading order in the $1 / L$ expansion [15], while the physical binding energy remains at a finite value at the infinite spatial volume limit. It requires huge computational cost to change the lattice spatial volume systematically. Second problem is a factorially large number of Wick contractions of quark-antiquark fields required for evaluation of 
the nucleus correlation functions: $\left\langle\mathscr{O}_{A}(t) \mathscr{O}_{A}^{\dagger}(0)\right\rangle^{t \gg 0} Z \exp (-M t)$. Suppose $\mathscr{O}_{A}$ represents a nucleus composed of $N_{p}$ protons and $N_{n}$ neutrons. A naive counting gives $\left(2 N_{p}+N_{n}\right) !\left(2 N_{n}+N_{p}\right)$ ! quark line diagrams after the Wick contractions of the up and down quark fields. This number quickly diverges beyond a three-nucleon system, e.g., 2880 for ${ }^{3} \mathrm{He}$ and 518400 for ${ }^{4} \mathrm{He}$. This problem, however, is partially tamed by considering equivalent contractions under permutation symmetry in terms of the protons or the neutrons in the operator. The computational cost can be reduced by avoiding the redundancy. In case of the ${ }^{4} \mathrm{He}$ nucleus, which consists of the same number of protons and neutrons, the artificial isospin symmetry $m_{\mathrm{u}}=m_{\mathrm{d}}$ also helps us reduce the necessary quark line diagrams. After all, it is found that only 1107 (93) contractions are required for the ${ }^{4} \mathrm{He}\left({ }^{3} \mathrm{He}\right)$ nucleus correlation function[12].

The ${ }^{4} \mathrm{He}$ nucleus has zero total angular momentum and positive parity $\mathrm{J}^{P}=0^{+}$with the isospin singlet $I=0$. The simplest ${ }^{4} \mathrm{He}$ operator is obtained by choosing the zero orbital angular momentum $L=0$, and hence $J=S$ with $S$ the total spin. Such an operator was already studied a long time ago[16]. Each nucleon in the sink operator is projected to have zero spatial momentum. An operator for ${ }^{3} \mathrm{He}$ nucleus, whose quantum numbers are $J^{P}=\frac{1}{2}^{+}, I=\frac{1}{2}$ and $I_{z}=\frac{1}{2}$, is constructed in a similar manner following the spin-isospin function given in Ref. [17].
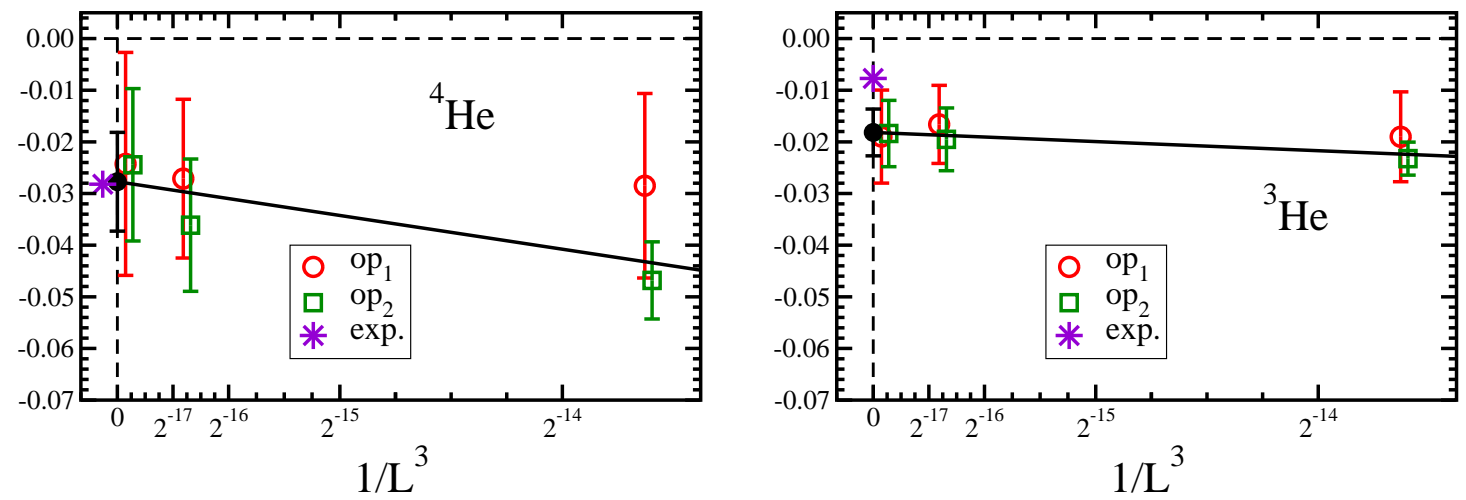

Figure 3: Spatial volume dependence of $-\Delta E_{L}=M-N_{N} m_{N}$ in $\mathrm{GeV}$ units for ${ }^{4} \mathrm{He}$ (left) and ${ }^{3} \mathrm{He}$ (right) nuclei with two types of source operators. Extrapolated results in the infinite spatial volume limit (filled circle) and experimental values (star) are also presented.

In order to determine the energy shift $\Delta E_{L}=N_{N} m_{N}-M$ precisely, the ratio of the He nucleus correlation function divided by the fourth power of the nucleon correlation function:

$$
R(t)=\frac{\left\langle\mathscr{O}_{\mathrm{He}}(t) \mathscr{O}_{\mathrm{He}}^{\dagger}(0)\right\rangle}{\left(\left\langle\mathscr{O}_{N}(t) \mathscr{O}_{N}^{\dagger}(0)\right\rangle\right)^{4}} \stackrel{t \gg 0}{\sim} Z \exp \left(\Delta E_{L} t\right)
$$

The energy shift is extracted by exponential fit of $R(t)$ in large $t$ region. Figure 3 shows the volume dependence of $\Delta E_{L}$ together with the combined error of the statistical and systematic ones as a function of $1 / L^{3}$. The measurements are performed with $L=24,48$ and 96 corresponding to 3.1, 6.1 and $12.3 \mathrm{fm}$, respectively. We observe little volume dependence for $\Delta E_{L}$ indicating a bound state, rather than the $1 / L^{3}$ dependence expected for a scattering state, for the ground state of $\mathrm{He}$ nuclei. 
The physical binding energy $\Delta E$ defined in the infinite spatial volume limit is extracted by a simultaneous fit of the data for two types of source operators employing a fit function of $\Delta E+C / L^{3}$ with $\Delta E$ and $C$ free parameters. The $1 / L^{3}$ term represents that $\Delta E_{L}$ may be contaminated by the scattering states, which are $O\left(1 / L^{3}\right)$ effects. The extrapolated result $\Delta E=27.7(9.6) \mathrm{MeV}$ for the ${ }^{4} \mathrm{He}$ nucleus agrees with the experimental value of $28.3 \mathrm{MeV}$. The consistency, however, is a little bit surprising because the calculation is performed at the unphysically heavy pion mass $m_{\pi}=0.8$ $\mathrm{GeV}$ which could considerably change a form of nuclear potential. For the ${ }^{3} \mathrm{He}$ nucleus the physical binding energy is $\Delta E=18.2(4.5) \mathrm{MeV}$, which is about twice larger than the experimental value of $7.72 \mathrm{MeV}$.

\section{Acknowledgments}

This work is supported in part by Grants-in-Aid for Scientific Research from the Ministry of Education, Culture, Sports, Science and Technology (Nos. 20105002, 22244018).

\section{References}

[1] H. Hamber and G. Parisi, Phys. Rev. Lett. 47, 1792 (1981).

[2] CP-PACS Collaboration, S. Aoki et al., Phys. Rev. Lett. 84, 238 (2000); Phys. Rev. D67, 034503 (2003).

[3] PACS-CS Collaboration, S. Aoki et al., Phys. Rev. D79, 034503 (2009).

[4] BMW Collaboration, S. Dürr et al., Science 322, 1224 (2008); ETM Collaboration, C. Alexandrou et al., Phys. Rev. D80, 114503 (2009).

[5] A. Duncan, E. Eichten, and H. Thacker, Phys. Rev. Lett. 76, 3894 (1996); RBC Collaboration, T. Blum et al., Phys. Rev. D76, 114508 (2007); T. Blum et al., Phys. Rev. D82, 094508 (2010); BMW Collaboration, A. Portelli et al., arXiv:1011.4189 [hep-lat].

[6] M. Lüscher, Nucl. Phys. B354, 531 (1991).

[7] CP-PACS Collaboration, S. Aoki et al., Phys. Rev. D76, 094506 (2007).

[8] QCDSF Collaboration, M. Göckeler et al., PoS LATTICE2008, 136 (2008); PACS-CS Collaboration, S. Aoki et al., PoS LATTICE2010, 108 (2010); ETM Collaboration, X. Feng, K. Jansen, and D. B. Renner, arXiv:1011.5288 [hep-lat]; BMW Collaboration, J. Frison et al., arXiv:1011.3413 [hep-lat].

[9] K. Rummukainen and S. Gottlieb, Nucl. Phys. B450, 397 (1995).

[10] M. Fukugita, Y. Kuramashi, H. Mino, M. Okawa, and A. Ukawa, Phys. Rev. D52, 3003 (1995).

[11] N. Ishii, S. Aoki, and T. Hatsuda, Phys. Rev. Lett. 99, 022001 (2007).

[12] PACS-CS Collaboration, Y. Yamazaki, Y. Kuramashi, and A. Ukawa, Phys. Rev. D81, 111504(R) (2010).

[13] CP-PACS Collaboration, S. Aoki et al., Phys. Rev. D71, 094504 (2005).

[14] S. Aoki, these proceedings.

[15] M. Lüscher, Commun. Math. Phys. 105, 153 (1986).

[16] J. E. Beam, Phys. Rev. 158, 907 (1967).

[17] M. Bolsterli and E. Jezak, Phys. Rev. 135, B510 (1964). 